\title{
Modelagem e propriedades termodinâmicas da secagem do epicarpo, mesocarpo e endocarpo do tucumã (Astrocaryum aculeatum)
}

\author{
Modelling and thermodynamic properties of the drying of \\ epicarp, mesocarp and endocarp of tucumã (Astrocaryum \\ aculeatum)
}

Tamyris Thaise Costa de Souza ${ }^{1 *} \oplus$, Evaldiney Ribeiro Monteiro ${ }^{2}$,
Cayck Trindade Ribeiro ${ }^{2}$, Diogo Silva de Souza ${ }^{2}$, Thamyres Torres dos Santos

${ }^{1}$ Universidade Federal do Sul e Sudeste do Pará (Unifesspa), Instituto de Geociências e Engenharias, Faculdade de Engenharia de Minas e Meio Ambiente, Marabá/PA - Brasil

${ }^{2}$ Universidade Federal do Sul e Sudeste do Pará (Unifesspa), Faculdade de Engenharia de Minas e Meio Ambiente (FEMMA), Marabá/PA - Brasil

${ }^{3}$ Universidade Federal do Sul e Sudeste do Pará (Unifesspa), Faculdade de Química, Marabá/PA - Brasil

*Corresponding Author: Tamyris Thaise Costa de Souza, Universidade Federal do Sul e Sudeste do Pará (Unifesspa), Instituto de Geociências e Engenharias, Faculdade de Engenharia de Minas e Meio Ambiente, Folha 17, Quadra 04, Lote Especial, s/n, Nova Marabá, CEP: 68505-080, Marabá/PA - Brasil, e-mail: ttcsouza@unifesspa.edu.br

Cite as: Souza, T. T. C., Monteiro, E. R., Ribeiro, C. T., Souza, D. S., \& Santos, T. T. (2021). Modelling and thermodynamic properties of the drying of epicarp, mesocarp and endocarp of tucumã (Astrocaryum aculeatum). Brazilian Journal of Food Technology, 24, e2020032. https://doi.org/10.1590/1981-6723.03220

\begin{abstract}
Resumo
O tucumã é um fruto amazônico, possui alto poder nutricional e é utilizado em diversos segmentos econômicos. Portanto, estudos para a redução de umidade devem ser realizados para o aumento do tempo de prateleira deste fruto. Este trabalho tem como objetivo realizar o estudo de secagem do epicarpo (casca), mesocarpo (polpa) e endocarpo (amêndoa) do tucumã nas temperaturas de $60^{\circ} \mathrm{C}, 70^{\circ} \mathrm{C}$ e $80^{\circ} \mathrm{C}$. Foi observado que o aumento da temperatura reduziu o tempo de queda da razão de umidade do epicarpo, mesocarpo e endocarpo. Modelos matemáticos foram utilizados para estimar dados experimentais e foram calculadas propriedades termodinâmicas do processo. Baseado no maior $\mathrm{R}^{2}$ e o menor SE e DQM, o modelo Logístico apresentou melhor ajuste para cinética de secagem dentre os avaliados, estimando energia de ativação de $39,50 \mathrm{~kJ} \mathrm{~mol}^{-1}, 46,62 \mathrm{~kJ} \mathrm{~mol}^{-1} \mathrm{e} \mathrm{17,76} \mathrm{kJ} \mathrm{mol}{ }^{-1}$ para o epicarpo, mesocarpo e endocarpo, respectivamente. Os resultados das propriedades termodinâmicas mostraram que a entalpia $(\Delta \mathrm{H})$ caracteriza a secagem como um processo endotérmico. A entropia $(\Delta \mathrm{S})$ diminui com o aumento da temperatura. Os valores da energia de Gibbs são positivos, ou seja, o processo é não espontâneo e necessita de energia externa para difusividade da água no ar.
\end{abstract}

Palavras-chave: Tucumã; Secagem; Razão de umidade; Modelagem matemática; Propriedades termodinâmicas; Alimentos. 


\begin{abstract}
Tucumã is an Amazonian fruit, has high nutritional value and is used in many economic segments. Therefore, studies to reduce the moisture of this products should be carried out to increase the shelf life of this fruit. This work aimed to study the drying of epicarp (shell), mesocarp (pulp) and endocarp (almond) of tucumã at 60,70 and $80^{\circ} \mathrm{C}$. It was observed that the increase in temperature has accelerated the reduction of moisture ratio in the epicarp, mesocarp and endocarp. Mathematical models were used to estimate experimental data as well as thermodynamic properties were calculated. Based on the highest $R^{2}$ and the lowest Standard Error (SE) and Mean Square Deviation (MSD), the Logistic model showed the best fit for drying kinetics among models evaluated. The activation energy results were $39.50 \mathrm{~kJ} \cdot \mathrm{mol}^{-1}, 46.62 \mathrm{~kJ} \mathrm{~mol}^{-1}$ and $17.76 \mathrm{~kJ} \mathrm{~mol}^{-1}$ for the epicarp, mesocarp and endocarp, respectively. The results of thermodynamic properties showed that enthalpy $(\Delta \mathrm{H})$ could characterize drying as an endothermic process. Entropy $(\Delta S)$ decreased with increasing temperature. The Gibbs energy values were positive, i.e., the process is characterized as non-spontaneous and need external energy in order to diffuse water molecules into the air.
\end{abstract}

Keywords: Tucumã; Drying; Ratio humidity; Mathematical modeling; Thermodynamic properties; Foods.

\title{
1 Introdução
}

A Amazônia apresenta uma grande diversidade de frutos de amplo potencial nutritivo de grande interesse social e econômico. Dessa forma, há interesse de diversas áreas para a utilização desse poder nutricional presente nesses frutos, dentre as quais, as áreas alimentícia, farmacêutica e cosmética. O tucumã (Astrocaryum aculeatum) é um fruto presente na região amazônica, muito consumido in natura e/ou subprodutos, como, por exemplo, recheios de sanduíches, cremes e sorvetes, devido ao seu potencial nutritivo (Carneiro et al., 2017; Santos et al., 2018). É indicado para alimentação humana e de animais domésticos, uma vez que o mesocarpo se caracteriza por uma fonte alimentícia altamente calórica, já que tem elevado conteúdo de lipídeos. Possui quantidades significativas do precursor da vitamina A (Yuyama et al., 2008), teores de fibras e vitamina E (Ribeiro \& Soares, 1995), além de ação antioxidante (Vieira et al., 2017).

A palmeira do tucumã floresce nos meses de maio a julho e frutifica de dezembro a maio, embora em algumas plantas possam ser encontrados frutos durante o ano inteiro (Oliveira et al., 2003, 2011). Como o seu cultivo se dá em uma região geográfica específica, isso pode dificultar a disponibilidade integral desse alimento e o acesso ao produto fresco. Dessa forma, vê-se aí a necessidade da redução do teor de umidade, aumentando a vida útil do fruto e então viabilizar a sua utilização comercial e industrial em locais mais distantes da região nativa. No entanto, ainda se encontram poucos trabalhos com o intuito de contribuir para a sua domesticação e aproveitamento, tendo a sua comercialização ainda caracterizada por um mercado local.

A secagem é um dos processos utilizados para a conservação dos alimentos e a redução do seu volume, facilitando assim armazenamento e transporte do produto (Celestino, 2010; Leonardi \& Azevedo, 2018). O processo de secagem baseia-se na redução hídrica presente nos frutos, o que consequentemente provoca a diminuição da atividade microbiana nesse produto, reduzindo assim perdas do produto e deterioração pós-colheita (Oliveira et al., 2015).

O secador de bandeja (ou de circulação de ar forçada) é um dos equipamentos mais utilizados na desidratação de alimentos. O seu funcionamento consiste em uma operação em batelada com passagem de ar quente sobre a superfície do sólido, além da sua construção ser relativamente barata (McCabe et al., 1993). O processo de secagem pode ser descrito por modelos matemáticos, buscando o tempo ideal para a redução do teor de água presente no produto, o que é importante para o armazenamento e para a projeção de equipamento de secagem. Além disso, são úteis para buscar melhorias para a eficiência da operação (Kadam \& Balasubramanian, 2011; Meziane, 2011; Sousa et al., 2011).

A determinação das propriedades termodinâmicas é importante para viabilizar a compreensão do processo, como, por exemplo, a variação de entalpia $(\Delta \mathrm{H})$, entropia $(\Delta \mathrm{S})$ e energia livre de Gibbs $(\Delta \mathrm{G})$. A variação de 
entalpia mensura a diferença de energia na interação das moléculas de água com o produto durante o processo (Silva et al., 2019). A variação de entropia está associada com o grau de desordem do sistema, é uma função de estado que tende a aumentar em sistema isolado (Corrêa et al., 2017). Além disso, a entropia pode estar relacionada com a ligação ou a repulsão das forças no sistema, e com o arranjo espacial água-produto (McMinn et al., 2005). A energia livre de Gibbs é a energia necessária para realizar a transferência das moléculas de água presente no produto para o ar, ou seja, é o trabalho realizado pelo sistema para realizar o processo de sorção (Thys et al., 2010).

Com base na importância e na necessidade da secagem de alimentos, o presente trabalho tem, como propósitos, secar o epicarpo (casca), mesocarpo (polpa) e endocarpo (amêndoa) do tucumã nas temperaturas de 60,70 e $80^{\circ} \mathrm{C}$ e avaliar o ajuste de diferentes modelos matemáticos aos dados obtidos experimentalmente. Os resultados obtidos pela modelagem serão utilizados para estimar as propriedades termodinâmicas do processo.

\section{Materiais e métodos}

O trabalho foi realizado no Laboratório de Tecnologia de Alimentos da Universidade Federal do Sul e Sudeste do Pará - Unifesspa, Unidade II, Campus Marabá, Pará. O fruto utilizado foi adquirido em Belém-PA, no Mercado Ver-o-Peso.

O tucumã foi inicialmente selecionado e lavado em água corrente, para a retirada das impurezas provenientes da colheita e dos frutos que estivessem aparentemente em um estado inadequado para o consumo. Na Figura 1, é mostrado o fluxograma metodológico, apresentando todas as etapas para o processo de secagem.

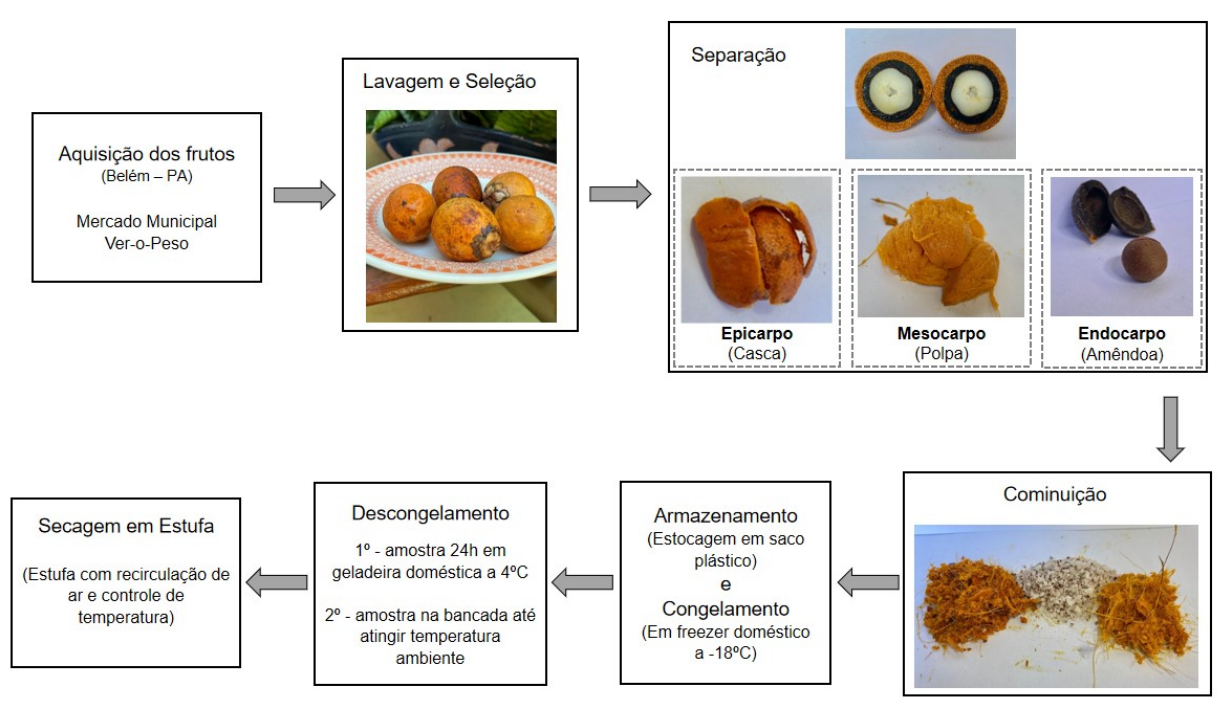

Figura 1. Fluxograma geral de preparação do mesocarpo, epicarpo e endocarpo do tucumã para o processo de secagem.

Após a lavagem e seleção dos frutos, foi realizada a etapa de separação, na qual, com auxílio de facas, foram separadas as três partes dos frutos: casca (epicarpo), polpa (mesocarpo) e amêndoa (endocarpo). Em seguida, essas partes foram trituradas (cominuição), utilizando um liquidificador doméstico, para melhorar o processo de secagem do material em estudo, pois o aumento da superfície de contato do material ajuda na difusão da água do produto para o ar. 
As amostras trituradas foram postas em sacos plásticos e armazenados em freezer a $-18{ }^{\circ} \mathrm{C}$, para evitar a perda do material. As secagens foram realizadas em triplicatas, utilizando placas de Petri. Para o descongelamento, o material foi transferindo para a geladeira a $4{ }^{\circ} \mathrm{C}$, durante $24 \mathrm{~h}$ e, em seguida, foi colocado na bancada do laboratório até que atingisse a temperatura média do ambiente $\left(25^{\circ} \mathrm{C}\right)$.

Para o processo de secagem, foi utilizada uma Estufa de Secagem. O equipamento se caracteriza como uma unidade de operação descontínua, usada para operações em pequena escala (Foust et al., 1982) e tem como base a remoção da água do alimento por aquecimento por circulação de ar (Quimis Q314M252). As amostras, separadas em placa de Petri, foram colocadas em bandejas. Esse processo foi realizado para o endocarpo, mesocarpo e epicarpo do fruto a $60{ }^{\circ} \mathrm{C}, 70{ }^{\circ} \mathrm{C}$ e $80^{\circ} \mathrm{C}$, até que o equilíbrio fosse atingido. Ou seja, o critério adotado para parar o processo de secagem foi a verificação de perda de massa (umidade) desprezível. A pesagem foi realizada em balança analítica (Even FA-2204B-BI) utilizando quantidades iniciais correspondentes a 9,75 $\mathrm{g}$ do epicarpo, $20 \mathrm{~g}$ do mesocarpo e $9,12 \mathrm{~g}$ do endocarpo.

A razão de umidade $\left(r_{u}\right)$ das amostras foi determinada utilizando a Equação 1.

$r_{u}=\frac{U^{t}-U_{e}}{U^{i}-U_{e}}$

Em que:

$U^{t}=$ teor de água presente na amostra no tempo $\mathrm{t}$ (g água/g da amostra seca);

$U_{e}=$ teor de água presente no equilíbrio;

$U^{i}=$ teor de umidade da amostra no tempo inicial (Oliveira et al., 2015).

Na Tabela 1, são mostrados os modelos utilizados para estimar os dados experimentais e avaliar o ajuste da cinética de secagem para o epicarpo, mesocarpo e endocarpo do tucumã (Equações 2, 3, 4 e 5).

Tabela 1. Modelos matemáticos utilizados para avaliação dos ajustes das curvas de cinética de secagem.

\begin{tabular}{ccc}
\hline Denominação & Modelo & Equação \\
\hline Verna & $r_{u}=\mathrm{a} \cdot e^{(-k t)}+(1-\mathrm{a}) \cdot e^{\left(-K_{1} t\right)}$ & $(2)$ \\
\hline Logaritmo & $r_{u}=\mathrm{a} \cdot e^{(-k t)}+c$ & $(3)$ \\
\hline Logístico & $r_{u}=\frac{\mathrm{a}}{\left(1+b \cdot e^{(k t)}\right)}$ & $(5)$ \\
\hline Aproximação da Difusão & $r_{u}=\mathrm{a} \cdot e^{(-k t)}+(1-\mathrm{a}) \cdot e^{(-k b t)}$ & $(4)$ \\
\hline
\end{tabular}

$\mathrm{r}_{\mathrm{u}}=$ razão de umidade; $\mathrm{k}=$ constante de secagem $\left(\mathrm{s}^{-1}\right) ; \mathrm{K}_{1}, \mathrm{a}, \mathrm{b}, \mathrm{c}=$ coeficientes dos modelos, que dependem na natureza do produto (adimensional); $\mathrm{t}=$ tempo de secagem (minutos).

Também serão avaliados os parâmetros de coeficiente de determinação $\left(R^{2}\right)$, desvio quadrático médio (DQM) (Equação 6) e o desvio-padrão da estimativa (SE) (Equação 7).

$$
\begin{aligned}
& D Q M=\left[\frac{1}{n} \sum_{i=1}^{n}(Y-\hat{Y})^{2}\right]^{\frac{1}{2}} \\
& S E=\left[\frac{\sum_{i=1}^{n}(Y-\hat{Y})^{2}}{G L R}\right]^{\frac{1}{2}}
\end{aligned}
$$

Em que:

$\mathrm{Y}=$ valor estimado experimentalmente; 
$\hat{\mathrm{Y}}=$ valor calculado pelo modelo;

GLR = graus de liberdade do modelo;

$\mathrm{n}=$ número de dados observados.

Quando se trata de um processo de secagem, a constante cinética do processo deve incluir a temperatura do material e os efeitos de concentração de umidade. A alta mobilidade das moléculas ocorre quando o teor de água é alto. Ademais, a dependência da temperatura é frequentemente expressa no tipo de taxa de reação química ou na forma da equação de Arrhenius (Equação 8), na qual k é definido como constante de secagem (Chen \& Mujumdar, 2008).

$k=k_{0} \exp \left(-\frac{E_{a}}{R T}\right)$

Em que:

$k_{0}=$ fator pré-exponencial $\left(\mathrm{s}^{-1}\right)$;

$R=$ constante universal dos gases $\left(8,314 \mathrm{~kJ} \mathrm{kmol}^{-1} \mathrm{~K}^{-1}\right)$;

$E_{a}$ a energia de ativação $\left(\mathrm{kJ} \mathrm{kmol}^{-1}\right)$;

$T$ = temperatura (K) (Oliveira et al., 2015).

Dessa forma, como o cálculo da constante $\mathrm{k}$ relaciona a redução de $\mathrm{r}_{\mathrm{u}}$ com o tempo do processo para diferentes considerações de temperatura de secagem (Oliveira et al., 2015), o valor de k pode ser utilizado para mensurar as propriedades termodinâmicas com base no modelo matemático de ajuste aos dados experimentais. Estudos mostram que a equação de Arrhenius descreve satisfatoriamente a relação da mobilidade de moléculas de água, no processo de secagem, com a temperatura (Faria et al., 2012; Horuz et al., 2018; Santos et al., 2019a; Silva et al., 2016).

As propriedades termodinâmicas - entalpia $(\Delta \mathrm{H})$, entropia $(\Delta \mathrm{S})$ e energia de Gibbs $(\Delta \mathrm{G})$ - são obtidas utilizando as Equações 9, 10 e 11, respectivamente. A determinação dessas propriedades foi pelo método descrito pela constante universal dos gases (Silva et al., 2019).

$\Delta H=E_{a}-R T$

$\Delta S=R\left[\ln \left(D_{O}\right)-\ln \left(\frac{k_{b}}{h_{p}}\right)-\ln T\right]$

$\Delta G=\Delta H-T \Delta S$

Em que:

$\Delta H=$ variação da entalpia específica $\left(\mathrm{J} \mathrm{mol}^{-1}\right)$;

$\Delta S=$ variação da entropia específica $\left(\mathrm{J} \mathrm{mol}^{-1} \mathrm{~K}^{-1}\right)$;

$\Delta G=$ energia livre de Gibbs $\left(\mathrm{J} \mathrm{mol}^{-1}\right)$;

$k_{b}=$ constante de Boltzmann, igual a $1,38 \times 10^{-23}\left(\mathrm{~J} \mathrm{~K}^{-1}\right)$;

$h_{p}=$ constante de Planck igual a $6,626 \times 10^{-34}\left(\mathrm{~J} \mathrm{~s}^{-1}\right)$;

$T$ = temperatura de secagem $(\mathrm{K})$.

\section{Resultados e discussão}

Para a validação do modelo matemático que melhor estima o processo físico de secagem, foram determinados (Tabela 2) o desvio padrão de estimativa (SE), o desvio quadrático médio (DQM) e o coeficiente de determinação $\left(\mathrm{R}^{2}\right)$. 
Tabela 2. Parâmetros dos modelos matemáticos, desvio-padrão da estimativa (SE), desvio quadrático médio (DQM) e coeficientes de determinação $\left(\mathrm{R}^{2}\right)$ relativos às curvas de cinética de secagem do epicarpo, mesocarpo e endocarpo do tucumã.

\begin{tabular}{|c|c|c|c|c|c|c|c|}
\hline \multirow[b]{2}{*}{ Modelo } & \multirow{2}{*}{$\begin{array}{l}\text { Parte do } \\
\text { Fruto }\end{array}$} & \multirow{2}{*}{$\begin{array}{l}\text { Temp. } \\
\left({ }^{\circ} \mathrm{C}\right)\end{array}$} & \multicolumn{2}{|c|}{ Parâmetros do modelo } & \multirow[b]{2}{*}{ SE } & \multirow[b]{2}{*}{ DQM } & \multirow[b]{2}{*}{$\mathbf{R}^{2}$} \\
\hline & & & Coeficientes do modelo & $\begin{array}{c}\text { Constante de secagem } \\
(\mathbf{k})\end{array}$ & & & \\
\hline \multirow{9}{*}{$\frac{5}{5}$} & \multirow{3}{*}{ Epicarpo } & 60 & $\mathrm{a}=-24,410 ; \mathrm{K}_{1}=1,1418$ & 1,1673 & 0,01579 & 0,01489 & 0,99900 \\
\hline & & 70 & $\mathrm{a}=23,251 ; \mathrm{K}_{1}=0,6635$ & 0,6782 & 0,03279 & 0,03089 & 0,99456 \\
\hline & & 80 & $\mathrm{a}=24,8321 ; \mathrm{K}_{1}=0,7608$ & 0,7766 & 0,02922 & 0,02754 & 0,99532 \\
\hline & \multirow{3}{*}{ Mesocarpo } & 60 & $a=-1,9108 ; K_{1}=0,3360$ & 0,3537 & 0,00318 & 0,00304 & 0,99996 \\
\hline & & 70 & $a=-26,114 ; K_{1}=0,8181$ & 0,8384 & 0,01983 & 0,01898 & 0,99838 \\
\hline & & 80 & $a=-15,276 ; K_{1}=0,7835$ & 0,80351 & 0,01039 & 0,00994 & 0,99949 \\
\hline & \multirow{3}{*}{ Endocarpo } & 60 & $a=19,165 ; K_{1}=1,0545$ & 1,0736 & 0,01884 & 0,01762 & 0,99811 \\
\hline & & 70 & $a=13,356 ; K_{1}=1,3907$ & 1,4237 & 0,01251 & 0,01170 & 0,99907 \\
\hline & & 80 & $\mathrm{a}=0,9539 ; \mathrm{K}_{1}=0,6755$ & 3,4691 & 0,00242 & 0,00226 & 0,99996 \\
\hline \multirow{9}{*}{ 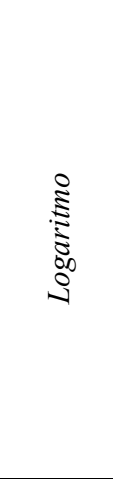 } & \multirow{3}{*}{ Epicarpo } & 60 & $a=1,0649 ; c=-0,0564$ & 0,6412 & 0,01687 & 0,01590 & 0,99886 \\
\hline & & 70 & $\mathrm{a}=1,0581 ; \mathrm{c}=-0,0300$ & 1,0608 & 0,03797 & 0,03579 & 0,99270 \\
\hline & & 80 & $a=1,0506 ; c=-0,0265$ & 1,2248 & 0,03575 & 0,03371 & 0,99299 \\
\hline & \multirow{3}{*}{ Mesocarpo } & 60 & $\mathrm{a}=1,0013 ; \mathrm{c}=-0,0021$ & 0,3032 & 0,00324 & 0,00310 & 0,99997 \\
\hline & & 70 & $\mathrm{a}=1,0579 ; \mathrm{c}=-0,0282$ & 0,4622 & 0,03226 & 0,03088 & 0,99573 \\
\hline & & 80 & $\mathrm{a}=1,0220 ; \mathrm{c}=-0,0143$ & 0,5383 & 0,01390 & 0,01331 & 0,99909 \\
\hline & \multirow{3}{*}{ Endocarpo } & 60 & $\mathrm{a}=1,0204 ; \mathrm{c}=-0,0097$ & 1,4820 & 0,02131 & 0,01993 & 0,99758 \\
\hline & & 70 & $\mathrm{a}=1,011 ; \mathrm{c}=-0,0067$ & 1,9109 & 0,01467 & 0,01372 & 0,99872 \\
\hline & & 80 & $\mathrm{a}=0,9914, \mathrm{c}=0,0083$ & 3,2422 & 0,00565 & 0,00529 & 0,99979 \\
\hline \multirow{9}{*}{ 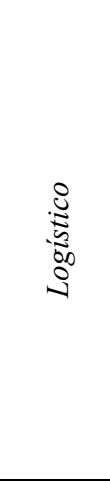 } & \multirow{3}{*}{ Epicarpo } & 60 & $\mathrm{a}=2,0139 ; \mathrm{b}=1,0328$ & 1,0219 & 0,01451 & 0,01368 & 0,99916 \\
\hline & & 70 & $a=1,4087 ; b=0,4172$ & 1,9984 & 0,01171 & 0,01103 & 0,99931 \\
\hline & & 80 & $a=1,4172 ; b=0,4207$ & 2,2809 & 0,01024 & 0,00965 & 0,99943 \\
\hline & \multirow{3}{*}{ Mesocarpo } & 60 & $a=23,464 ; b=22,5223$ & 0,3123 & 0,00312 & 0,00298 & 0,99997 \\
\hline & & 70 & $a=1,5784 ; b=0,6062$ & 0,7850 & 0,01606 & 0,01538 & 0,99894 \\
\hline & & 80 & $a=3,1201 ; b=2,1453$ & 0,6791 & 0,01041 & 0,00996 & 0,99949 \\
\hline & \multirow{3}{*}{ Endocarpo } & 60 & $a=1,935 ; b=0,9318$ & 2,1863 & 0,00840 & 0,00786 & 0,99962 \\
\hline & & 70 & $a=2,2606 ; b=1,2599$ & 2,6024 & 0,00820 & 0,00767 & 0,99960 \\
\hline & & 80 & $a=5,7 \times 10^{14} ; b=5,71_{x} 10^{14}$ & 3,1445 & 0,00902 & 0,00843 & 0,99945 \\
\hline \multirow{9}{*}{ 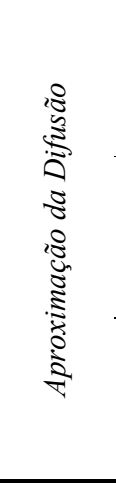 } & \multirow{3}{*}{ Epicarpo } & 60 & $a=0 ; b=1$ & 0,7180 & 0,02985 & 0,02814 & 0,99643 \\
\hline & & 70 & $a=0 ; b=1$ & 1,1116 & 0,04397 & 0,04146 & 0,99020 \\
\hline & & 80 & $a=0 ; b=1$ & 1,2810 & 0,04115 & 0,03879 & 0,99071 \\
\hline & \multirow{3}{*}{ Mesocarpo } & 60 & $a=0 ; b=1$ & 0,3047 & 0,00338 & 0,00324 & 0,99996 \\
\hline & & 70 & $a=0 ; b=1$ & 0,4725 & 0,03821 & 0,03658 & 0,99401 \\
\hline & & 80 & $a=0 ; b=1$ & 0,5502 & 0,01663 & 0,01592 & 0,99870 \\
\hline & \multirow{3}{*}{ Endocarpo } & 60 & $a=0 ; b=1$ & 1,5080 & 0,02272 & 0,02126 & 0,99725 \\
\hline & & 70 & $a=0 ; b=1$ & 1,9421 & 0,01560 & 0,01459 & 0,99856 \\
\hline & & 80 & $a=0 ; b=1$ & 3,1457 & 0,00902 & 0,08441 & 0,99945 \\
\hline
\end{tabular}

$\mathrm{k}=$ constantes de secagem $\left(\mathrm{s}^{-1}\right) ; \mathrm{K}_{1}, \mathrm{a}, \mathrm{b}, \mathrm{c}=$ coeficientes dos modelos; Temp. = Temperatura; $\mathrm{SE}=$ desvio padrão de estimativa; $\mathrm{DQM}=$ Desvio Quadrático Médio; $\mathrm{R}^{2}=$ coeficiente de determinação. 
Observando os dados da Tabela 2, verificou-se que o maior valor de SE chega a 0,04397. Dessa forma, esses valores mostram que existe boa aproximação do valor calculado pelos modelos aos dados experimentais, já que quando valores de desvio $<10 \%$ ocorrem, isso significa um ajuste satisfatório dos modelos para representação de secagem (Madamba et al., 1996; Mohapatra \& Srinivasa Rao, 2005). Porém, têm-se que os menores valores de SE foram obtidos para o modelo Logístico. Os resultados do DQM são analisados similarmente ao SE. Nesse caso, o modelo Logístico apresentou menores resultados de DQM, atestando maior proximidade entre os dados experimentais e os dados calculados de razão de umidade.

Em geral, valores de $\mathrm{R}^{2}$ acima de 0,98 são considerados elevados e, quando altos valores de $\mathrm{R}^{2}$ estão associados a baixos resultados de desvio médio, tem-se um ajuste adequado entre os dados teóricos e experimentais (Côrrea Filho et al., 2015; Meneghetti et al., 2012; Murthy \& Manohar, 2014). Os modelos aplicados, em todas as condições de secagem $\left(60^{\circ} \mathrm{C}, 70^{\circ} \mathrm{C}\right.$ e $\left.80^{\circ} \mathrm{C}\right)$, tiveram resultados de $\mathrm{R}^{2}$ satisfatórios, variando entre 0,99020 e 0,99997 .

No entanto, avaliando a combinação dos resultados de SE, DQM e $\mathrm{R}^{2}$, o modelo mais adequado para descrever esse processo de secagem foi o Logístico com os seguintes resultados: (a) Epicarpo (casca) - nas temperaturas de $60{ }^{\circ} \mathrm{C}, 70{ }^{\circ} \mathrm{C}$ e $80^{\circ} \mathrm{C}$, os coeficientes de determinação foram 0,99916 , 0,99931 e 0,99943 , respectivamente. Além disso, esse modelo apresentou baixos valores de SE, iguais a $0,01451,0,01171$ e 0,01024, e baixos resultados de DQM, iguais a 0,01368, 0,01103 e 0,00965 a $60{ }^{\circ} \mathrm{C}, 70{ }^{\circ} \mathrm{C}$ e $80{ }^{\circ} \mathrm{C}$, respectivamente; (b) Mesocarpo (polpa) - os valores de SE e DQM foram de 0,00318 e 0,00304 a $60{ }^{\circ} \mathrm{C}, 0,01606$ e 0,01538 a $70{ }^{\circ} \mathrm{C}$, e 0,01041 e 0,00996 a $80{ }^{\circ} \mathrm{C}$, respectivamente; consequentemente, tem melhor ajuste do modelo, para condições de secagem; (c) Endocarpo (amêndoa) - os parâmetros estatísticos obtidos para o modelo Logístico, a $60{ }^{\circ} \mathrm{C}$, foram, $0,99962\left(\mathrm{R}^{2}\right), 0,00840(\mathrm{SE})$ e $0,00786(\mathrm{DQM})$; a $70{ }^{\circ} \mathrm{C}$, foram, $0,99960\left(\mathrm{R}^{2}\right), 0,00820(\mathrm{SE})$ e 0,00767 (DQM), e a $80{ }^{\circ} \mathrm{C}$, foram, $0,99945\left(\mathrm{R}^{2}\right), 0,00902$ (SE) e 0,00843 (DQM), correspondendo ao conjunto de dados que classifica esse modelo como o que melhor se ajusta aos dados experimentais nas temperaturas citadas. Na Tabela 2, observa-se que a constante de secagem $\mathrm{k}$ aumentou com a elevação da temperatura, indicando que a taxa de secagem é proporcional à elevação da temperatura do ar (Araujo et al., 2017).

No estudo de secagem de frutos de espinheiro secos e arroz, o modelo logístico obteve ajuste satisfatório aos dados experimentais, com base nos dados de coeficiente de correlação, erro padrão e desvio quadrático médio. Apesar de o modelo Logístico, nesses estudos, não ser eleito o mais adequado, a justificativa dos autores para escolha do modelo de melhor ajuste se dá por verificação do maior $\mathrm{R}^{2}$ e os menores SE e DQM (Amiri Chayjan et al., 2015; Cihan et al., 2007). Então, para a secagem do tucumã, o modelo logístico apresenta melhor capacidade de estimativa da cinética de secagem dentre os avaliados.

A Figura 2 mostra os dados experimentais de razão de umidade $\left(r_{u}\right)$ em função do tempo para o epicarpo, mesocarpo e endocarpo do tucumã a $60^{\circ} \mathrm{C}, 70^{\circ} \mathrm{C}$ e $80^{\circ} \mathrm{C}$, e as curvas do modelo matemático de melhor ajuste.

Analisando-se a Figura 2, observa-se que, durante o processo de secagem, no que tange à razão de umidade $\left(\mathrm{r}_{\mathrm{u}}\right)$ para o epicarpo, mesocarpo e endocarpo, em menor temperatura $\left(60^{\circ} \mathrm{C}\right)$, foi necessário um maior tempo de processo para atingir o $r_{u}$ de equilíbrio $\left(r_{u e}\right)$ em relação as demais condições de secagem. Isso pode ser explicado devido à pressão de vapor das moléculas de água presente no fruto aumentar em função da temperatura, favorecendo o processo de difusão da água no ar (Oliveira et al., 2015). É observado um aumento da inclinação das curvas de secagem à medida que a temperatura aumenta. Isso ocorre porque, no processo realizado em temperaturas mais altas, existe mais calor transferido do meio aquecido para o material e, então, o deslocamento da água do interior do material para a sua superfície é maior (Castro et al., 2016; Reis et al., 2013). 
Nesse caso, as análises experimentais mostraram os resultados de umidade em base seca ( $\left.\mathrm{U}_{\mathrm{b.s}}\right)$, após o processo de secagem atingir o equilíbrio de: (a) Epicarpo - 2,5 horas de secagem para alcançar o $\mathrm{r}_{\text {ue }}$ nas temperaturas de $70{ }^{\circ} \mathrm{C}$ e $80{ }^{\circ} \mathrm{C}$, com valores em $\mathrm{U}_{\mathrm{b} . \mathrm{s}}$ de $0,029 \mathrm{~g} / \mathrm{g}$ e $0,01 \mathrm{~g} / \mathrm{g}$, respectivamente. A $60{ }^{\circ} \mathrm{C}$, a umidade (b.s) atingiu o valor de 0,002 $\mathrm{g} / \mathrm{g}$ em 4,5 horas; (b) Mesocarpo - o $\mathrm{r}_{\text {ue }}$ apareceu após 8,5 horas a $70{ }^{\circ} \mathrm{C}$ e $80{ }^{\circ} \mathrm{C}$, com $\mathrm{U}_{\mathrm{b} . \mathrm{s}}$ de $0,002 \mathrm{~g} / \mathrm{g}$ e $0,0015 \mathrm{~g} / \mathrm{g}$, respectivamente. No entanto, a $60{ }^{\circ} \mathrm{C}$, a umidade chegou a 0,0004 g/g em 20,5 horas; (c) Endocarpo - 2,0 horas foi o tempo de secagem observado para atingir o equilíbrio a $70{ }^{\circ} \mathrm{C}$ e $80{ }^{\circ} \mathrm{C}$, com $\mathrm{U}_{\mathrm{b} . \mathrm{s}}$ de $0,012 \mathrm{~g} / \mathrm{g}$ e $0,0030 \mathrm{~g} / \mathrm{g}$, respectivamente. Já no processo a $60{ }^{\circ} \mathrm{C}$, o resultado foi de $0,004 \mathrm{~g} / \mathrm{g}$ em 2,5 horas.

Os valores de umidade no equilíbrio mostram que o mesocarpo alcança resultados menores em um tempo superior comparado ao epicarpo e ao endocarpo. Isso ocorre, possivelmente, devido à maior quantidade de água presente na polpa, como também à maior energia de ativação, o que resulta em uma menor difusividade por unidade de tempo.

Na Tabela 3, são apresentados os valores de energia de ativação $\left(E_{a}\right)$ e as propriedades termodinâmicas entalpia $(\Delta \mathrm{H})$, entropia $(\Delta \mathrm{S})$ e energia livre de Gibbs $(\Delta \mathrm{G})$ - do processo de secagem para o epicarpo, mesocarpo e endocarpo do tucumã a $60{ }^{\circ} \mathrm{C}, 70{ }^{\circ} \mathrm{C}$ e $80^{\circ} \mathrm{C}$.

Tabela 3. Resultados de energia de ativação $\left(E_{a}\right)$, entalpia $(\Delta H)$, entropia $(\Delta S)$ e energia livre de Gibbs $(\Delta G)$, para o processo de secagem do epicarpo, mesocarpo e endocarpo.

\begin{tabular}{|c|c|c|c|c|c|}
\hline \multirow[b]{2}{*}{ Parte do fruto } & \multirow{2}{*}{$\begin{array}{c}\mathbf{E}_{\mathrm{a}} \\
\left(\mathrm{kJ} \mathrm{mol}^{-1}\right)\end{array}$} & \multirow[b]{2}{*}{$\begin{array}{c}\text { Temperatura } \\
\left({ }^{\circ} \mathrm{C}\right)\end{array}$} & \multicolumn{3}{|c|}{ Parâmetros Termodinâmicos } \\
\hline & & & $\begin{array}{c}\Delta \mathbf{H} \\
\left(\mathrm{kJ} \mathrm{mol}^{-1}\right)\end{array}$ & $\begin{array}{c}\Delta S \\
\left(\mathrm{~kJ} \mathrm{~mol}^{-1} \mathrm{~K}^{-1}\right)\end{array}$ & $\begin{array}{c}\Delta \mathbf{G} \\
\left(\mathrm{kJ} \mathrm{mol}^{-1}\right)\end{array}$ \\
\hline \multirow{3}{*}{ Epicarpo } & \multirow{3}{*}{39,50} & 60 & 36,7328 & $-0,1264$ & 78,8295 \\
\hline & & 70 & 36,6496 & $-0,1266$ & 80,0944 \\
\hline & & 80 & 36,5665 & $-0,1269$ & 81,3618 \\
\hline \multirow{3}{*}{ Mesocarpo } & \multirow{3}{*}{46,61} & 60 & 43,8471 & $-0,1144$ & 81,9529 \\
\hline & & 70 & 43,7639 & $-0,1146$ & 83,0981 \\
\hline & & 80 & 43,6808 & $-0,1149$ & 84,2456 \\
\hline \multirow{3}{*}{ Endocarpo } & \multirow{3}{*}{17,76} & 60 & 14,9892 & $-0,1860$ & 76,9652 \\
\hline & & 70 & 14,9052 & $-0,1863$ & 78,8269 \\
\hline & & 80 & 14,8220 & $-0,1865$ & 80,6911 \\
\hline
\end{tabular}

Os resultados de energia de ativação $\left(\mathrm{E}_{\mathrm{a}}\right)$, mostrados na Tabela 3 , foram estimados com base no modelo Logístico. Os valores de $\mathrm{E}_{\mathrm{a}}$ estão relacionados à energia necessária para que ocorra o processo de difusão da água (Martins et al., 2015). Os produtos agrícolas apresentam uma variação dessa energia de 12,7 a $110 \mathrm{~kJ}$ $\mathrm{mol}^{-1}$ (Zogzas et al., 1996). No epicarpo e endocarpo do tucumã, a $E_{\mathrm{a}}$ foi de $39,5 \mathrm{~kJ} \mathrm{~mol}^{-1}$ e $22,51 \mathrm{~kJ} \mathrm{~mol}^{-1}$, respectivamente. Estes valores são superiores aos resultados apontados na secagem do acuri, 17,66 kJ mol-1 (Santos et al., 2019b), e inferiores aos resultados apresentados para a secagem do arroz, 51,03 kJ mol ${ }^{-1}$ (Corrêa et al., 2017). Para o mesocarpo, a energia determinada foi de $46,61 \mathrm{~kJ} \mathrm{~mol}^{-1}$, ou seja, a polpa do tucumã necessita de mais energia para ser seca, quando comparado às outras partes do fruto. Analisando-se os valores de $\mathrm{E}_{\mathrm{a}}$, podemos dizer que a maior difusividade da água é encontrada no endocarpo e a menor, no mesocarpo, já que quanto menor a energia de ativação, maior será a difusividade por unidade de tempo, pois a energia de ativação é considerada uma energia limite a ser atingida para que o processo de difusão no produto possa acontecer (Kashaninejad et al., 2007; Silva et al., 2015). Altos valores de $\mathrm{E}_{\mathrm{a}}$ também podem estar associados à forte ligação da água ao produto; consequentemente, sua remoção é conduzida pela estrutura da amostra (Amiri Chayjan et al., 2015).

Analisando-se os dados mostrados na Tabela 3, é observada uma redução da entalpia $(\Delta \mathrm{H})$ com o aumento da temperatura. $\Delta \mathrm{H}$ nos indica a energia que é necessária para remoção da água associada à matéria no processo de secagem (Guimarães et al., 2018; Oliveira et al., 2010). Os processos que apresentam valores de 
variação de entalpia menores indicam uma menor quantidade de energia para a secagem do produto (Oliveira et al., 2016). A pressão parcial do vapor de água da amostra é aumentada devido à elevação da temperatura do ar de secagem, porém a pressão parcial do ar não é alterada. Assim, a difusão de água do interior para a superfície do produto é mais rápida e a consequente perda da água por dessorção, em todas as partes estudadas, também aumenta. Portanto, o aumento da temperatura resultou em uma menor quantidade de energia necessária para o processo de perda de água por difusão. Análise semelhante para a entalpia em processo de secagem foi observada no estudo de secagem de polpa de [patauá] (Santos et al., 2019a) e de okara (Guimarães et al., 2018). Temos que a $\Delta \mathrm{H}_{\text {mesocarpo }}>\Delta \mathrm{H}_{\text {epicarpo }}>\Delta \mathrm{H}_{\text {endocarpo, ou seja, a polpa do tucumã }}$ é a parte do fruto que mais precisa de energia, uma vez que a entalpia varia de 43,6808 a 43,8471 kJ mol ${ }^{-1}$ $\mathrm{K}^{-1}$. Dessa forma, a maior umidade presente no mesocarpo faz com que seja necessária maior quantidade de energia a ser absorvida pelo sistema. O processo é endotérmico, pois $\Delta H$ é positivo, caracterizando a absorção de calor durante a operação (Shafaei et al., 2016). A secagem de folhas de boldo é um processo endotérmico com $\Delta \mathrm{H}$ positivos, variando de $15,5329 \mathrm{~kJ} \mathrm{~mol}^{-1}\left(20^{\circ} \mathrm{C}\right), 15,3667 \mathrm{~kJ} \mathrm{~mol}^{-1}\left(40^{\circ} \mathrm{C}\right) \mathrm{e} 15,2004 \mathrm{~kJ} \mathrm{~mol}^{-1}(60$ ${ }^{\circ} \mathrm{C}$ ) (Silva et al., 2019). Na secagem de polpa de patauá, os valores de $\Delta \mathrm{H}$ foram $37,5008 \mathrm{~kJ} \mathrm{~mol}^{-1}\left(40^{\circ} \mathrm{C}\right.$ ), $37,4177 \mathrm{~kJ} \mathrm{~mol}^{-1}\left(50{ }^{\circ} \mathrm{C}\right)$ e $37,3345 \mathrm{~kJ} \mathrm{~mol}^{-1}\left(60^{\circ} \mathrm{C}\right.$ ) (Santos et al., 2019a). Porém, na secagem de morangos, o processo é exotérmico, já que os valores de $\Delta \mathrm{H}$ foram de $-481,21 \mathrm{~J} \mathrm{~mol}^{-1}\left(40^{\circ} \mathrm{C}\right),-564,35 \mathrm{~J} \mathrm{~mol}^{-1}\left(50^{\circ} \mathrm{C}\right)$ e - $647,49 \mathrm{~J} \mathrm{~mol}^{-1}\left(60^{\circ} \mathrm{C}\right)$ (Oliveira et al., 2015; Silva et al., 2019).
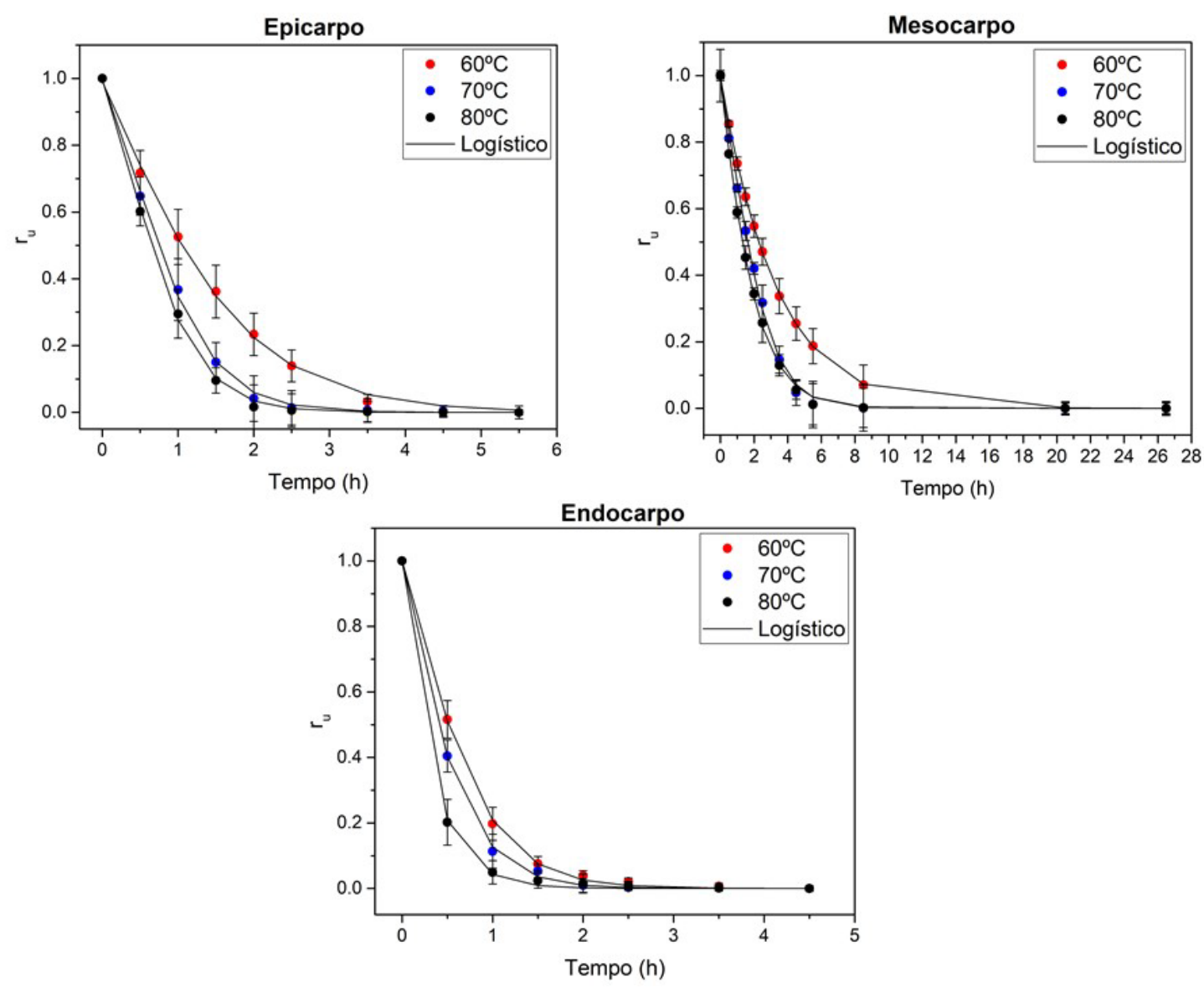

Figura 2. Curvas de secagem experimental e estimadas pelo modelo Logístico para o epicarpo, mesocarpo e endocarpo do tucumã, nas temperaturas de $60^{\circ} \mathrm{C}, 70^{\circ} \mathrm{C}$ e $80^{\circ} \mathrm{C}$.

Os resultados de entropia $(\Delta \mathrm{S})$ diminuem com o aumento da temperatura. $\mathrm{O}$ aumento na pressão parcial de vapor da água no produto devido ao aumento da temperatura do ar de secagem também provocou maior excitação das moléculas e abaixamento da viscosidade da água em temperaturas mais altas. Esses fatores 
possivelmente levam ao aumento da velocidade do processo de difusão de água, à redução dos sítios disponíveis e à restrição dos movimentos das moléculas de água, uma vez que se tem redução de umidade e, consequentemente, redução da entropia no processo. Os valores de $\Delta \mathrm{S}$ negativos podem estar ligados à existência de distorção química e/ou modificações estruturais do produto durante o processo de secagem (Cagnin et al., 2017; Corrêa et al., 2010; Corrêa et al., 2011; Moreira et al., 2008). Nas secagens de pimenta cabacinha, os valores da variação de entropia foram $-0,29575 \mathrm{~kJ} \mathrm{~mol}^{-1} \mathrm{~K}^{-1}\left(60{ }^{\circ} \mathrm{C}\right),-0,29599 \mathrm{~kJ} \mathrm{~mol}^{-1} \mathrm{~K}^{-1}(70$ $\left.{ }^{\circ} \mathrm{C}\right),-0,29623 \mathrm{~kJ} \mathrm{~mol}^{-1} \mathrm{~K}^{-1}\left(80{ }^{\circ} \mathrm{C}\right),-0,29646 \mathrm{~kJ} \mathrm{~mol}^{-1} \mathrm{~K}^{-1}\left(90{ }^{\circ} \mathrm{C}\right)$ e $-0,29669 \mathrm{~kJ} \mathrm{~mol}^{-1} \mathrm{~K}^{-1}\left(100{ }^{\circ} \mathrm{C}\right)$ (Silva et al., 2016). Para a secagem das sementes de chia, os resultados encontrados para a variação de entropia foram de $-0,21505 \mathrm{~kJ} \mathrm{~mol}^{-1} \mathrm{~K}^{-1},-0,21531 \mathrm{~kJ} \mathrm{~mol}^{-1} \mathrm{~K}^{-1} \mathrm{e}-0,21557 \mathrm{~kJ} \mathrm{~mol}^{-1} \mathrm{~K}^{-1}$, e para a secagem de polpa de bacaba, foram de $-0,3039 \mathrm{~kJ} \mathrm{~mol}^{-1} \mathrm{~K}^{-1},-0,304 \mathrm{~kJ} \mathrm{~mol}^{-1} \mathrm{~K}^{-1}$ e $-0,3044 \mathrm{~kJ} \mathrm{~mol}^{-1} \mathrm{~K}^{-1}$, nas temperaturas de $40{ }^{\circ} \mathrm{C}, 50{ }^{\circ} \mathrm{C}$ e $60^{\circ} \mathrm{C}$, respectivamente (Morais et al., 2019; Oliveira et al., 2016).

Os valores de $\Delta \mathrm{G}$ para o epicarpo, mesocarpo e endocarpo são positivos, o que nos justifica o processo de secagem como não espontâneo, ou seja, é necessária uma energia externa para a desidratação, caracterizando a existência de uma reação endergônica (Guimarães et al., 2018; Oliveira et al., 2016; Silva et al., 2016). O $\Delta \mathrm{G}$ do epicarpo, mesocarpo e endocarpo cresce com o aumento da temperatura do processo de secagem. Esse resultado pode ser discutido através da relação da energia livre de Gibbs, em pressão e temperatura constantes, com o trabalho necessário para tornar os sítios de sorção disponíveis, ou seja, é uma função termodinâmica responsável por quantificar a máxima energia liberada em um determinado processo (Nkolo Meze'e et al., 2008). Então, é possível afirmar que, na secagem do tucumã, o trabalho útil gerado nesse processo aumenta à medida que a temperatura aumenta.

Para o epicarpo, os valores de $\Delta \mathrm{G}$ foram de $78,8295 \mathrm{~kJ} \mathrm{~mol}^{-1}\left(60^{\circ} \mathrm{C}\right), 80,0944 \mathrm{~kJ} \mathrm{~mol}^{-1}\left(70{ }^{\circ} \mathrm{C}\right)$ e 81,3618 $\mathrm{kJ} \mathrm{mol}^{-1}\left(80^{\circ} \mathrm{C}\right)$, e para endocarpo, foram de $76,9652 \mathrm{~kJ} \mathrm{~mol}^{-1}\left(60^{\circ} \mathrm{C}\right), 78,8299 \mathrm{~kJ} \mathrm{~mol}^{-1}\left(70{ }^{\circ} \mathrm{C}\right)$ e 80,6911 $\mathrm{kJ} \mathrm{mol}^{-1}\left(80^{\circ} \mathrm{C}\right.$ ). Os resultados de energia livre de Gibbs na secagem da polpa (mesocarpo) foram de 81,9529 $\mathrm{kJ} \mathrm{mol}^{-1}, 83,0981 \mathrm{~kJ} \mathrm{~mol}^{-1}$ e $84,2456 \mathrm{~kJ} \mathrm{~mol}^{-1}$, nas mesmas condições de secagem. Na secagem do acuri, o $\Delta \mathrm{G}$ foi de $139,489 \mathrm{~kJ} \mathrm{~mol}^{-1}\left(60^{\circ} \mathrm{C}\right), 143,230 \mathrm{~kJ} \mathrm{~mol}^{-1}\left(70{ }^{\circ} \mathrm{C}\right), 146,974 \mathrm{~kJ} \mathrm{~mol}^{-1}\left(80^{\circ} \mathrm{C}\right)$ e $150,720 \mathrm{~kJ} \mathrm{~mol}^{-1}$ $\left(90^{\circ} \mathrm{C}\right)$ (Santos et al., 2019b).

\section{Conclusão}

No estudo de secagem e determinação das propriedades termodinâmicas do tucumã foi observado que diferentes temperaturas resultam em tempos de secagem distintos, em que a maior temperatura favoreceu o processo de difusão da água presente no produto para o ar.

Os valores de umidade de equilíbrio mostraram que o mesocarpo alcança resultados menores em comparação ao epicarpo e ao endocarpo, porém em um tempo mais longo. Possivelmente, tal fato é devido à maior energia de ativação, o que resulta em uma menor difusividade por unidade de tempo.

Com base nos maiores valores de $\mathrm{R}^{2}$ e menores resultados de SE e DQM, o modelo Logístico foi o que teve melhor ajuste aos dados experimentais, sendo assim usado para estimar energia de ativação e propriedades termodinâmicas.

Os resultados positivos de entalpia $(\Delta \mathrm{H})$ qualificam o processo como endotérmico. A difusão de água do interior do produto é mais rápida em temperaturas mais altas e, assim, uma menor quantidade de energia é necessária para o processo. As variações de entropia $(\Delta \mathrm{S})$ do processo são diminuídas por restrição dos movimentos das moléculas de água, uma vez que se tem mais rápida redução de umidade com o aumento da temperatura. A energia livre de Gibbs $(\Delta \mathrm{G})$ positiva demonstra que o processo é não espontâneo e depende de energia externa para a difusividade da água no ar. 


\section{Referências}

Amiri Chayjan, R., Kaveh, M., \& Khayati, S. (2015). Modeling drying characteristics of hawthorn fruit under microwaveconvective conditions. Journal of Food Processing and Preservation, 39(3), 239-253. http://dx.doi.org/10.1111/jfpp.12226

Araujo, W., Goneli, A. L. D., Corrêa, P. C., Hartmann, C. P. F., \& Martins, E. A. S. (2017). Modelagem matemática da secagem dos frutos de amendoim em camada delgada. Ciência Agronômica, 48(3), 448-457.

Cagnin, C., Lima, M. S., Silva, R. M., Silva, M. A. P., Plácido, G. R., Freitas, B. S. M., \& Oliveira, D. E. C. (2017). Garlic: Kinetic drying and thermodynamic properties. Bioscience Journal, 33(4), 905-913.

Carneiro, A. B. A., Pinto, E. J. S., Ribeiro, I. F., Magalhães, M. R. G., \& Monteiro Neto, M. A. B. (2017). Efeito da Astrocaryum aculeatum (Tucumã) na toxicidade da Doxorrubicina: Modelo experimental in vivo. Acta Paulista de Enfermagem, 30(3), 233239. http://dx.doi.org/10.1590/1982-0194201700036

Castro, D., Melo Silva, L., Moreira, I., Sousa, F., Silva, W., \& Almeida, F. (2016). Análise e descrição matemática da secagem de polpa de taturubá (Pouteriamacrophyllas (Lam.) Eyma.) em camada fina. Engevista, 18(2), 309.

http://dx.doi.org/10.22409/engevista.v18i2.734

Celestino, S. M. C. (2010). Princípios de secagem de alimentos. Embrapa.

Chen, X. D., \& Mujumdar, A. S. (2008). Drying technologies in food processing (1st ed.). Chichester: John Wiley \& Sons.

Cihan, A., Kahveci, K., \& Hacıhafızoğlu, O. (2007). Modelling of intermittent drying of thin layer rough rice. Journal of Food Engineering, 79(1), 293-298. http://dx.doi.org/10.1016/j.jfoodeng.2006.01.057

Côrrea Filho, L. C., Andrade, E. T., Martinazzo, A. P., D’Andrea, E. M., Sousa, F. A., \& Figueira, V. G. (2015). Cinética de secagem, contração volumétrica e análise da difusão líquida do figo (Ficus carica L.). Revista Brasileira de Engenharia Agrícola e Ambiental, 19(8), 797-802. http://dx.doi.org/10.1590/1807-1929/agriambi.v19n8p797-802

Corrêa, P. C., Botelho, F. M., Oliveira, G. H. H., Goneli, A. L. D., Resende, O., \& Campos, S. C. (2011). Mathematical modeling of the drying process of corn ears. Acta Scientiarum. Agronomy, 33(1), 575-581.

$\mathrm{http}: / / \mathrm{dx}$.doi.org/10.4025/actasciagron.v33i4.7079

Corrêa, P. C., Oliveira, G. H. H., Botelho, F. M., Goneli, A. L. D., \& Carvalho, F. M. (2010). Modelagem matemática e determinação das propriedades termodinâmicas do café (Coffea arabica L.) durante o processo de secagem. Revista Ceres, 57(5), 595-601. http://dx.doi.org/10.1590/S0034-737X2010000500005

Corrêa, P. C., Oliviera, G. H. H., Oliveira, A. P. L. R., Botelho, F. M., \& Goneli, A. L. D. (2017). Thermodynamic properties of drying process and water absorption of rice grains. CYTA: Journal of Food, 15(2), 204-210. http://dx.doi.org/10.1080/19476337.2016.1238012

Faria, R. Q., Teixeira, I. R., Devilla, I. A., Ascheri, D. P. R., \& Resende, O. (2012). Cinética de secagem de sementes de crambe. Revista Brasileira Agrícola e Ambiental, 16(5), 573-583. http://dx.doi.org/10.1590/S1415-43662012000500014 Foust, A. S., Wenzel, L. A., Clump, C. W., Maus, L., \& Andersen, L. B. (1982). Princípio das operações unitárias. Rio de Janeiro: Guanabara Dois.

Guimarães, R. M., Oliveira, D. E. C., Resende, O., Silva, J. S., Rezende, T. A. M., \& Egea, M. B. (2018). Thermodynamic properties and drying kinetics of "okara.". Revista Brasileira de Engenharia Agrícola e Ambiental, 22(6), 418-423. http://dx.doi.org/10.1590/1807-1929/agriambi.v22n6p418-423

Horuz, E., Bozkurt, H., Karataş, H., \& Maskan, M. (2018). Simultaneous application of microwave energy and hot air to whole drying process of apple slices: Drying kinetics, modeling, temperature profile and energy aspect. Heat and Mass Transfer, 54(2), 425-436. http://dx.doi.org/10.1007/s00231-017-2152-y

Kadam, D. M., \& Balasubramanian, S. (2011). Foam mat drying of tomato juice. Journal of Food Processing and Preservation, 35(4), 488-495. http://dx.doi.org/10.1111/j.1745-4549.2010.00492.x

Kashaninejad, M., Mortazavi, A., Safekordi, A., \& Tabil, L. G. (2007). Thin-layer drying characteristics and modeling of pistachio nuts. Journal of Food Engineering, 78(1), 98-108. http://dx.doi.org/10.1016/j.jfoodeng.2005.09.007

Leonardi, J. G., \& Azevedo, B. M. (2018). Métodos de conservação de alimentos. Revista Saúde em Foco, 10(1), 1-11.

Madamba, P. S., Driscoll, R. H., \& Buckle, K. A. (1996). The thin-layer drying characteristics of garlic slices. Journal of Food Engineering, 29(1), 75-97. http://dx.doi.org/10.1016/0260-8774(95)00062-3

Martins, E. A. S., Lage, E. Z., Goneli, A. L. D., Hartmann Filho, C. P., \& Lopes, J. G. (2015). Cinética de secagem de folhas de timbó (Serjania marginata Casar). Revista Brasileira de Engenharia Agrícola e Ambiental, 19(3), 238-244. http://dx.doi.org/10.1590/1807-1929/agriambi.v19n3p238-244

McCabe, W. L., Smith, J. C., \& Harriott, P. (1993). Unit operations of chemical engineering (5th ed.). New York: McGraw-Hill. McMinn, W. A. M., Al-Muhtaseb, A. H., \& Magee, T. R. A. (2005). Enthalpy-entropy compensation in sorption phenomena of starch materials. Food Research International, 38(5), 505-510. http://dx.doi.org/10.1016/j.foodres.2004.11.004

Meneghetti, V. L., Aosani, E., Rocha, J. C., Oliveira, M., Elias, M. C., \& Pohndorf, R. S. (2012). Modelos matemáticos para a secagem intermitente de arroz em casca. Revista Brasileira de Engenharia Agrícola e Ambiental, 16(10), 1115-1120. http://dx.doi.org/10.1590/S1415-43662012001000012

Meziane, S. (2011). Drying kinetics of olive pomace in a fluidized bed dryer. Energy Conversion and Management, 52(3), 16441649. http://dx.doi.org/10.1016/j.enconman.2010.10.027

Mohapatra, D., \& Srinivasa Rao, P. (2005). A thin layer drying model of parboiled wheat. Journal of Food Engineering, 66(4), 513-518. http://dx.doi.org/10.1016/j.jfoodeng.2004.04.023 
Morais, M. F. S., Santos, J. R. O., Santos, M. P., Santos, D. C., Costa, T. N., \& Lima, J. B. (2019). Modeling and thermodynamic properties of "bacaba" pulp drying. Revista Brasileira de Engenharia Agrícola e Ambiental e Ambiental, 23(9), 702-708. http://dx.doi.org/10.1590/1807-1929/agriambi.v23n9p702-708

Moreira, R., Chenlo, F., Torres, M. D., \& Vallejo, N. (2008). Thermodynamic analysis of experimental sorption isotherms of loquat and quince fruits. Journal of Food Engineering, 88(4), 514-521. http://dx.doi.org/10.1016/j.jfoodeng.2008.03.011

Murthy, T. P. K., \& Manohar, B. (2014). Hot air drying characteristics of mango ginger: Prediction of drying kinetics by mathematical modeling and artificial neural network. Journal of Food Science and Technology, 51(12), 3712-3721. PMid:25477637. http://dx.doi.org/10.1007/s13197-013-0941-y

Nkolo Meze'e, Y. N., Noah Ngamveng, J., \& Bardet, S. (2008). Effect of enthalpy-entropy compensation during sorption of water vapour in tropical woods: The case of Bubinga (Guibourtia Tessmanii J. Léonard; G. Pellegriniana J.L.). Thermochimica Acta, 468(1), 1-5. http://dx.doi.org/10.1016/j.tca.2007.11.002

Oliveira, G. H. H., Aragão, D. M. S., Oliveira, A. P. L. R., Silva, M. G., \& Gusmão, A. C. (2015). Modelagem e propriedades termodinâmicas na secagem de morangos. Brazilian Journal of Food Technology, 18(4), 314-321. http://dx.doi.org/10.1590/1981-6723.5315

Oliveira, G. H. H., Corrêa, P. C., Araújo, E. F., Valente, D. S. M., \& Botelho, F. M. (2010). Desorption isotherms and thermodynamic properties of sweet corn cultivars (Zea mays L.). International Journal of Food Science \& Technology, 45(3), 546-554. http://dx.doi.org/10.1111/j.1365-2621.2009.02163.x

Oliveira, G. H. H., Costa, M. R., Botelho, F. M., Viana, J. L., \& Garcia, T. R. B. (2016). Thermodynamic properties and kinetics of drying process of chia seeds (Salvia hispanica L). Research Journal of Seed Science, 9(2), 36-41. http://dx.doi.org/10.3923/rjss.2016.36.41

Oliveira, M. S. P., Couturier, G., \& Beserra, P. (2003). Biologia da polinizaçãoda palmeira tucumã (Astrocaryum vulgare Mart.) em Belém, Pará, Brasil. Acta Botanica Brasílica, 17(3), 343-353. http://dx.doi.org/10.1590/S0102-33062003000300002

Oliveira, M. S. P., Oliveira, N. P., \& Abreu, L. F. (2011). Estabelecimento de área de coleta de sementes de tucumã-do-pará (Comunicado Técnico, No. 225, pp. 1-5). Belém: Embrapa.

Reis, R. C., Côrrea, P. C., Devilla, I. A., Santos, E. S., Ascheri, D. P. R., Servulo, A. C. O., \& Souza, A. B. M. (2013). Drying of yam starch (Discorea ssp.) and glycerol filmogenic solutions at different temperatures. Lebensmittel-Wissenschaft + Technologie, 50(2), 651-656. http://dx.doi.org/10.1016/j.Iwt.2012.07.033

Ribeiro, C. C., \& Soares, M. S. (1995). Caracterização do fruto e elaboração de geléia da polpa de tucumã (Astrocaryum vulgare Marte). In Encontro Regional do Norte e Nordeste (213 p.). Campinas: Sociedade Brasileira de Ciência e Tecnologia de Alimentos.

Santos, D. C., Costa, T. N., Franco, F. B., Castro, R. C., Ferreira, J. P. L., Souza, M. A. S., \& Santos, J. C. P. (2019a). Cinética de secagem e propriedades termodinâmicas da polpa de patauá (Oenocarpus bataua Mart.). Brazilian Journal of Food Technology, 22, 1-11. http://dx.doi.org/10.1590/1981-6723.30518

Santos, D. C., Leite, D. D. F., Lisbôa, J. F., Ferreira, J. P. L., Santos, F. S., Lima, T. L. B., Figueiredo, R. M. F., \& Costa, T. N. (2019b). Modelagem e propriedades termodinâmicas da secagem de fatias de acuri. Brazilian Journal of Food Technology, 22, e2018031. http://dx.doi.org/10.1590/1981-6723.03118

Santos, M. M. R., Fernandes, D. S., Cândido, C. J., Cavalheiro, L. F., Silva, A. F., Nascimento, V. A., Ramos Filho, M. M., Santos, E. F., \& Hiane, P. A. (2018). Physical-chemical, nutritional and antioxidant properties of tucumã (Astrocaryum huaimi Mart.) fruits. Semina: Ciências Agrárias, 39(4), 1517-1532. http://dx.doi.org/10.5433/1679-0359.2018v39n4p1517

Shafaei, S. M., Masoumi, A. A., \& Roshan, H. (2016). Analysis of water absorption of bean and chickpea during soaking using Peleg model. Journal of the Saudi Society of Agricultural Sciences, 15(2), 135-144. http://dx.doi.org/10.1016/j.jssas.2014.08.003

Silva, H. W., Rodovalho, R. S., Velasco, M. F., Silva, C. F., \& Vale, L. S. R. (2016). Kinetics and thermodynamic properties related to the drying of "Cabacinha" pepper fruits. Revista Brasileira de Engenharia Agrícola e Ambiental, 20(2), 174-180. http://dx.doi.org/10.1590/1807-1929/agriambi.v20n2p174-180

Silva, L. A., Resende, O., Virgolino, Z. Z., Bessa, J. F. V., Morais, W. A., \& Vidal, V. M. (2015). Cinética de secagem e difusividade efetiva em folhas de jenipapo (Genipa americana L.). Revista Brasileira de Plantas Medicinais, 17(4 Supl 2), 953963. http://dx.doi.org/10.1590/1983-084X/14_106

Silva, N. C. B., Santos, S. G. F., Silva, D. P., Silva, I. L., \& Rodovalho, R. S. (2019). Drying kinetics and thermodynamic properties of boldo leaves (Plectranthus barbatus Andrews). Journal of Agrarian Sciences, 47(1), 1-7. http://dx.doi.org/10.15361/1984-5529.2019v47n1p01-07

Sousa, K. A., Resende, O., Chaves, T. H., \& Costa, L. M. (2011). Cinética de secagem do nabo forrageiro (Raphanus sativus L.). Ciência Agronômica, 42(4), 883-892. http://dx.doi.org/10.1590/S1806-66902011000400009

Thys, R. C. S., Noreña, C. P. Z., Marczak, L. D. F., Aires, A. G., \& Cladera-Olivera, F. (2010). Adsorption isotherms of pinhão (Araucaria angustifolia seeds) starch and thermodynamic analysis. Journal of Food Engineering, 100(3), 468-473. http://dx.doi.org/10.1016/j.jfoodeng.2010.04.033

Vieira, L. M., Azevedo, S. C. M., Silva, G. F., \& Albuquerque, P. M. (2017). Estudo do potencial antioxidante da polpa do tucumã (Astrocaryum aculeatum) in natura em embalagens à vácuo. The Journal of Engineering and Exact Sciences, 3(4), 672677. http://dx.doi.org/10.18540/24469416030420170672

Yuyama, L. K. O., Maeda, R. N., Pantoja, L., Aguiar, J. P. L., \& Marinho, H. A. (2008). Processamento e avaliação da vida-deprateleira do tucumã (Astrocaryum aculeatum Meyer) desidratado e pulverizado. Food Science and Technology, 28(2), 408412. http://dx.doi.org/10.1590/S0101-20612008000200021 
Modelagem e propriedades termodinâmicas da secagem do epicarpo, mesocarpo e endocarpo do tucumã (Astrocaryum aculeatum) Souza, T. T. C. et al.

Zogzas, N. P., Maroulis, Z. B., \& Marinos-Kouris, D. (1996). Moisture diffusivity data compilation in foodstuffs. Drying Technology, 14(10), 2225-2253. http://dx.doi.org/10.1080/07373939608917205

Financiamento: Nenhum.

Received: Feb. 11, 2020; Accepted: Apr. 19, 2021 